\title{
Density profile of a strictly two-dimensional Bose gas at finite temperature
}

\author{
K. K. Rajagopal, P. Vignolo, and M. P. Tosi* \\ INFM and Classe di Scienze, \\ Scuola Normale Superiore,I-56126 Pisa, Italy
}

\begin{abstract}
We study a Bose-condensed gas at finite temperature, in which the particles of the condensate and of the thermal cloud are constrained to move in a plane under radial harmonic confinement and interact via strictly two-dimensional collisions. The coupling parameters are obtained from a calculation of the many-body T-matrix and decreases as temperature increases through a dependence on the chemical potential and on the occupancy of excited states. We discuss the consequences on the condensate fraction and on the density profiles of the condensed and thermal components as functions of temperature, within a simplified form of the two-fluid model.
\end{abstract}

Key words: Bose gases, low-dimensional, density profiles PACS: 03.75.Fi, 05.30.Jp, 32.80.Pj

\section{Introduction}

Recent experiments have realized quasi two-dimensional (2D) cold Bose systems by tuning the anisotropy of the trapping potential $[1,2]$ and have stimulated interest in studying Bose-Einstein condensation in a low-dimensional regime. An important difference between the macroscopic $2 \mathrm{D}$ fluid and the 3D one is that at finite temperature phase fluctuations in 2D destroy the longrange order, in agreement with the Mermin-Wagner theorem [3]. A KosterlitzThouless transition to a superfluid state still occurs on cooling through the binding of vortices of opposite vorticity [4] and leads to an algebraic decay of the one-body density matrix. However, in a trapped 2D fluid the modifications of the density of states caused by the confining potential allow a true condensate to exist even at finite temperature.

\footnotetext{
* Corresponding author, e-mail: vignolo@sns.it
} 
Another important consequence of lowered dimensionality is that the T-matrix for two-body collisions in vacuo at low momenta and energy, which should be used to obtain the collisional coupling parameters to lowest order in the particle density, vanishes in the strictly $2 \mathrm{D}$ limit as the $s$-wave scattering length becomes larger than the width of the axial trapping $[5,6]$. It is then necessary to evaluate the scattering processes between pairs of Bose particles by taking into account the presence of a condensate and a thermal cloud through a many-body T-matrix formalism [7-9].

This formalism has already been used in a number of studies of low dimensional Bose gases, dealing in particular with phase fluctuations and the KosterlitzThouless transition in a variational approach $[7,8,10,11]$, with a mean-field evaluation of the breathing-mode frequency in a trapped 1D gas [12], and with the equilibrium density profile of a $2 \mathrm{D}$ condensate $[9,13]$. In the limit of zero temperature the condensate-condensate coupling parameter has been related to the two-body $\mathrm{T}$ matrix by considering that, when two particles in the condensate collide at zero momentum, they both require an energy equal to the chemical potential $\mu$ to be excited out of the condensate [9]. With increasing temperature the population of the excited states becomes non-negligible and a microscopic theory which also takes into account the depletion of the condensate is required [14]. An additional coupling parameter to describe the scattering processes between an atom in the condensate and an atom in the thermal cloud has been introduced by Stoof and co-workers [10] through the two-body T-matrix at energy $-\mu$.

The present paper is the finite-temperature extension of the work by Tanatar et al. [13], who studied the cross-over from a $3 \mathrm{D}$ to a $2 \mathrm{D}$ regime in the equilibrium density profiles at zero temperature. Here we limit ourselves to the strictly 2D case, where the scattering length has become larger than the axial thickness of the cloud. We give a semi-analytical expression for the many-body T-matrix elements corresponding to the condensate-condensate and to the condensatethermal cloud coupling parameters ( $g_{2}$ and $g_{1}$, respectively) and we use them in a two-fluid model [15] to evaluate the condensate fraction and the density profiles of the bosonic gas at increasing temperature. Congruently with the work of Tanatar et al. [13] we focus on the experimental parameter of Görlitz et al. [1] for the case of largest anisotropy.

The paper is organized as follows. Section. 2 summarizes the general treatment of the many-body T-matrix for two-body scattering processes in the presence of a condensate and a thermal cloud. In Sec. 3 we evaluate a simplified form of the two-fluid model for a 2D Bose gas at finite temperature. Finally, we present and discuss our results in Sec. 4. 


\section{Coupling parameters in a mixture of condensed and thermal bosons}

The scattering processes between pairs of atoms in a gas consisting of a BoseEinstein condensate and a thermal cloud are described by the many-body T-matrix $T^{M B}(E)$ as a function of the energy $E$. The coupling parameters are given by the matrix elements $\left\langle\mathbf{k}^{\prime}\left|T^{M B}(E)\right| \mathbf{k}\right\rangle \equiv T^{M B}\left(\mathbf{k}, \mathbf{k}^{\prime}, \mathbf{K} ; E\right)$, taken in the limit of zero energy and momenta. Here $\mathbf{k}$ and $\mathbf{k}^{\prime}$ are the incoming and outgoing relative momenta of the pair of center-of-mass momentum $\mathbf{K}$. We shall consider only the condensate-condensate and condensate-thermal cloud couplings and neglect the scattering between thermally excited atoms in the present case of a dilute gas.

Before discussing the many-body T-matrix, however, we shall first recall the behavior of the two-body T-matrix that describes collisions between pairs of particles in vacuo. We shall then discuss how the two T-matrices are related in the appropriate limit for a two-fluid system.

\subsection{The two-body T matrix}

The two-body T-matrix is the solution of the Lippmann-Schwinger equation,

$$
\begin{aligned}
\left\langle\mathbf{k}^{\prime}\left|T^{2 B}(\bar{E})\right| \mathbf{k}\right\rangle & =\left\langle\mathbf{k}^{\prime}\left|V\left(\left|\mathbf{r}_{1}-\mathbf{r}_{2}\right|\right)\right| \mathbf{k}\right\rangle \\
& +\sum_{\mathbf{q}}\left\langle\mathbf{k}^{\prime}\left|V\left(\left|\mathbf{r}_{1}-\mathbf{r}_{2}\right|\right)\right| \mathbf{q}\right\rangle \frac{1}{\bar{E}-2 \epsilon_{\mathbf{q}}}\left\langle\mathbf{q}\left|T^{2 B}(\bar{E})\right| \mathbf{k}\right\rangle,
\end{aligned}
$$

with $V\left(\left|\mathbf{r}_{1}-\mathbf{r}_{\mathbf{2}}\right|\right)$ being the interparticle potential. The center-of-mass energy for the pair of atoms is $\bar{E}$ and each atom of mass $m$ has single-particle excitation energy $\epsilon_{\mathbf{q}}=\hbar^{2} q^{2} / 2 m$, as collision takes place in free space. In Eq. (1) the collision is described by a single-loop interaction between the two atoms plus contributions involving all possible transition routes from state $|\mathbf{k}\rangle$ to state $\left|\mathbf{k}^{\prime}\right\rangle$ via intermediate states $|\mathbf{q}\rangle$.

In the case of hard-disk potential of strength $V_{0}=4 \pi \hbar^{2} / \mathrm{m}$ and in the dilute limit $\mathbf{k} a, \mathbf{k}^{\prime} a \ll 1$ where $a$ is the $2 \mathrm{D}$ scattering length, the solution of Eq. (1) is $[10]$

$$
\left\langle\mathbf{k}^{\prime}\left|T^{2 B}(\bar{E})\right| \mathbf{k}\right\rangle \approx \frac{4 \pi \hbar^{2} / m}{\ln \left|4 \hbar^{2} / \bar{E} m a^{2}\right|} .
$$

In this case the T-matrix is independent of the momenta, but depends on the logarithm of the energy and vanishes as $\bar{E}$ approaches zero. Therefore, the presence of the surrounding gas must be taken into account in the collisional processes. Following the proposal of Morgan et al. [16], this is done by setting 
$\bar{E}=-2 \mu$ for the mutual scattering of two condensate particles, this being the energy required for them to be excited out of the condensate. By a similar argument Al Khawaja et al. [10] set $\bar{E}=-\mu$ for the scattering between a boson in the condensate and a boson in the thermal cloud.

\subsection{The many-body T-matrix}

According to the above argument, a first approximation for the many-body T-matrix at zero momenta and energy is

$$
T_{n}^{M B}(0,0,0 ; 0)=T^{2 B}(0,0,0 ;-n \mu)
$$

with $n=1$ for condensate-thermal cloud scattering and $n=2$ for condensatecondensate scattering. Further many-body effects can enter the scattering processes attended by the presence of a condensate from attributing a Bogoliubov spectrum to the intermediate states [7]. At low momenta the many-body Tmatrix is the solution of the integral equation,

$$
\begin{aligned}
\left\langle\mathbf{k}^{\prime}\left|T^{M B}(E)\right| \mathbf{k}\right\rangle= & \left\langle\mathbf{k}^{\prime}\left|V\left(\left|\mathbf{r}_{\mathbf{1}}-\mathbf{r}_{\mathbf{2}}\right|\right)\right| \mathbf{k}\right\rangle+\sum_{\mathbf{q}}\left\langle\mathbf{k}^{\prime}\left|\mathbf{V}\left(\left|\mathbf{r}_{\mathbf{1}}-\mathbf{r}_{\mathbf{2}}\right|\right)\right| \mathbf{q}\right\rangle \\
& \times \frac{1+2 N\left(\omega_{\mathbf{q}}\right)}{E-2 \hbar \omega_{\mathbf{q}}}\left\langle\mathbf{q}\left|T^{M B}(E)\right| \mathbf{k}\right\rangle
\end{aligned}
$$

where $\hbar \omega_{\mathbf{q}} \approx \epsilon_{\mathbf{q}}+\mu$ are the Bogoliubov excitation energies in the Hatree approximation $\mu \ll \epsilon_{\mathbf{q}}$ and $N\left(\omega_{\mathbf{q}}\right) \approx\left\{\exp \left[\beta\left(\epsilon_{\mathbf{q}}+\mu\right)\right]-1\right\}^{-1}$ are the corresponding population factors. For a contact interaction potential Eq. (4) yields

$$
T^{M B}(0,0,0 ; E)=\left[\frac{1}{V_{0}}-\sum_{\mathbf{q}} \frac{1+2 N\left(\omega_{\mathbf{q}}\right)}{E-2 \hbar \omega_{\mathbf{q}}}\right]^{-1} .
$$

As discussed by Stoof and Bijlsma [7,8], Eq. (5) is still affected by an ultraviolet divergence, which can be remedied by replacing $V_{0}$ in favor of the two-body T-matrix. The final result is

$$
T_{n}^{M B}(0,0,0 ; 0)=T^{2 B}(0,0,0 ;-n \mu)\left[1+T^{2 B}(0,0,0 ;-n \mu) \sum_{\mathbf{q}} \frac{N\left(\omega_{\mathbf{q}}\right)}{\hbar \omega_{\mathbf{q}}}\right]^{-1},
$$

where $T^{2 B}(0,0,0 ;-n \mu)$ is given by Eq. (2) with $\bar{E}=-n \mu$.

\subsection{Calculation of coupling parameters}

The sum in the RHS of Eq. (6) can be evaluated analytically by replacing the sum over intermediate states by an integral over momentum. Setting $N\left(\omega_{\mathbf{q}}\right)=$ 
$\sum_{s=1}^{\infty} \exp \left(-s \beta \hbar \omega_{\mathbf{q}}\right)$, we get

$$
\sum_{q} \frac{N\left(\omega_{q}\right)}{\hbar \omega_{q}}=-\frac{m}{2 \pi \hbar^{2}} \sum_{s=1}^{\infty} E i(-s \beta \mu)
$$

where $\operatorname{Ei}(x)$ is the exponent-integral function. In the asymptotic low-temperature regime $(\beta \mu \gg 1)$ this thermal-population term can be approximated by

$$
\sum_{s=1}^{\infty} E i(-s \beta \mu) \rightarrow(\beta \mu)^{-1} \ln [1-\exp (-\beta \mu)]
$$

A numerical illustration is given in Fig. 1 for values of the system parameters appropriate to the experiments on ${ }^{23} \mathrm{Na}$ by Görlitz et al. [1] (see Sec. 4).

In summary, the coupling parameters in the 2D Bose gas are given by

$$
g_{n}^{M B}=\frac{4 \pi \hbar^{2} / m}{\ln \left|4 \hbar^{2} /\left(n m \mu a^{2}\right)\right|-2 \sum_{s=1}^{\infty} E i(-s \beta \mu)},
$$

with $n=2$ for collisions between pairs of condensate atoms and $n=1$ for collisions between an atom in the condensate and a thermally excited atom. These parameters depend on temperature both through the chemical potential and through the excited-state population factor given in Eq. (7) and asymptotically approximated by Eq. (8). If this population factor is dropped, one obtains from Eq. (3) the corresponding "two-body" coupling parameters as

$$
g_{n}^{2 B}=\frac{4 \pi \hbar^{2} / m}{\ln \left|4 \hbar^{2} /\left(n m \mu a^{2}\right)\right|} .
$$

In the next Section we evaluate the chemical potential and hence the coupling parameters in Eqs. (9) and (10) through a self-consistent evaluation of the density profiles in a two fluid-model.

\section{Equilibrium properties in the two-fluid model}

Let $n_{c}(r)$ and $n_{n c}(r)$ be the particle density profiles for the condensate and for the thermal cloud in a 2D Bose gas which is radially confined inside a isotropic planar trap described by the external potential $V_{\text {ext }}(r)=m \omega^{2} r^{2} / 2$. The twofluid model $[15,17]$ combines a solution of the Gross-Pitaevskii equation for the condensate with a Hatree-Fock model of the thermal cloud, which is treated as an ideal gas subject to an effective potential $V_{\text {eff }}(r)$. In the present case,

$$
V_{e f f}(r)=V_{e x t}(r)+2 g_{1} n_{c} .
$$

As already noted, we are neglecting the collisions between pairs of bosons belonging to the thermal cloud. It is well known that a full numerical solution 
of the Gross Pitaevskii equation can be avoided when the kinetic energy term in it can be neglected [18]. This yields the condensate density in the ThomasFermi approximation as

$$
n_{c}(r)=\left(1 / g_{2}\right)\left[\mu-V_{\text {ext }}(r)-2 g_{1} n_{n c}(r)\right] \theta\left(\mu-V_{\text {ext }}(r)-2 g_{1} n_{n c}(r)\right) .
$$

Since we are interested in examining qualitative behaviors rather than in attaining high numerical accuracy, we have adopted Eq. (12) for the condensate density and found that discontinuities occurring in the density profiles at the Thomas-Fermi radius can be eliminated by the simple expedient of introducing momentum cut-off in the expression for $n_{n c}(r)$. That is, we calculate $n_{n c}(r)$ from

$$
n_{n c}(r)=-\frac{m}{2 \pi \hbar^{2} \beta} \ln \left\{1-\exp \left[\beta\left(\mu-V_{e f f}(r)-\frac{p_{0}^{2}}{m}\right)\right]\right\},
$$

where we take $p_{0}=\sqrt{2 m g_{1} n_{n c}}[19]$. The model is then evaluated by solving self-consistently Eqs. (11)-(13) together with the condition that the areal integral of $n_{c}(r)+n_{n c}(r)$ should be equal to the total number $N$ of particles.

Before turning to a presentation of our numerical results, let us point out the wide range of validity of the present model. A mean-field treatment is valid when the diluteness condition $n_{c} a^{2} \ll 1$ holds and if the temperature of gas is outside the critical region. With regard to the thermal cloud, no significant differences have been found between the predictions of the Hatree-Fock and Popov approximations in the regime $n_{c} a^{2} \ll 1$, except at very low temperature where the thermal cloud is becoming negligible [20].

\section{Results and discussion}

For a numerical illustration, we have taken the values of particle number, the radial trap frequency, and the scattering length as appropriate for ${ }^{23} \mathrm{Na}$ atoms in the experiment of Görlitz et al. [1] $\left(N=5 \times 10^{5}, \omega=188.4 \mathrm{~Hz}, a=2.8\right.$ $\mathrm{nm})$. Whereas in their experiment collisions are in a 3D regime, we focus on a strictly $2 \mathrm{D}$ regime that could be reached experimentally by increasing either the trap anisotropy parameter or the scattering length.

In Fig. 2 we compare the temperature dependence of the coupling parameters $g_{n}^{2 B}$ (long dashes for $n=2$ and short dashes for $n=1$ ) with that of the coupling parameters $g_{n}^{M B}$ (full and dotted lines, respectively). It is evident that the many-body screening of the interactions due to the occupancy of excited states is quite large and rapidly increasing with temperature.

Such many-body screening has, however, very little effect on equillibrium properties of the gas for our choice of system parameters. Figure 3 reports the 
condensate fraction $N_{0} / N$ for the $g_{n}^{M B}$ model as a function of temperature, in comparison with that of an ideal Bose gas at the same values of the system parameters. As is well known, the transition temperature and the condensate fraction are lowered by the interactions. However, the $g_{n}^{2 B}$ model gives results that are practically the same as the $g_{n}^{M B}$ one.

Finally, the panels in Fig. 4 show the evolution of the density profiles for the condensate and for the thermal cloud with increasing temperature from near absolute zero to the critical temperature $T_{c} \simeq 0.95 T_{0}$ with $T_{0}=(\sqrt{6} / \pi) \hbar \omega \sqrt{N}$ being the critical temperature of the ideal gas [21]. The results in Fig. 4(a) are in good agreement with those of Tanatar et al. [13], except that the tails of the profile are missed in the Thomas-Fermi approximation. Again the screening of collision from the occupancy of the excited states is very small and becomes barely visible at $T \approx 0.75 T_{0}$.

In conclusion, the use of Eq. (3) to describe the many-body effects in twobody scattering processes in 2D Bose-condensed gas appears to be very good in regard to equillibrium properties at large values of particle number. A decrease in the number of particles lowers the chemical potential and may lead to observable effects for $N \approx 10^{3}$.

\section{Acknowledgements}

This work was partially supported by INFM under the project PRA-Photonmatter. Two of us (K.K.R. and M.P.T.) wish to thank the Condensed Matter Section of the Abdus Salam International Center for Theoretical Physics for their hospitality during the preparation of this work.

\section{References}

[1] A. Görlitz, J. M. Vogels, A. E. Leanhardt, C. Raman, T. L. Gustavson, J. R. Abo-Shaeer, A. P. Chikkatur, S. Gupta, S. Inouye, T. Rosenband, W. Ketterle, Phys. Rev. Lett. 87 (2001) 130402.

[2] A. I. Safonov, S. A. Vasilyev, I. S. Yasnikov, I. I. Lukashevich, S. Jaakkola, Phys. Rev. Lett. 81 (1998) 4545.

[3] N. D. Mermin, H. Wagner, Phys. Rev. Lett. 17 (1966) 1133.

[4] J. M. Kosterlitz, D. J. Thouless, J. Phys. C 6 (1973) 1181.

[5] M. Schick, Phys. Rev. A. 3 (1971) 1067. 
[6] V. N. Popov, Functional Integrals in Quantum Field Theory and Statistical Physics, (Reidel, Dordrecht 1983), Chap. 6.

[7] H. T. C. Stoof, M. Bijlsma, Phys. Rev. E 47 (1993) 939.

[8] M. Bijlsma, H. T. C. Stoof, Phys. Rev. A. 55 (1997) 498.

[9] M. D. Lee, S. A. Morgan, M. J. Davis, K. Burnett, Phys. Rev. A. 65 (2002) 043617.

[10] U. Al Khawaja, J. O. Andersen, N. P. Proukakis, H. T. C. Stoof, Phys. Rev. A. 66 (2002) 013615.

[11] U. Al Khawaja, J. O. Andersen, N. P. Proukakis, H. T. C. Stoof, Phys. Rev. A. 66 (2002) 059902.

[12] N. P. Proukakis, S. A. Morgan, S. Choi, K. Burnett, Phys. Rev. A. 58 (1998) 2435.

[13] B. Tanatar, A. Minguzzi, P. Vignolo, M. P. Tosi, Phys. Lett. A 302 (2002) 131.

[14] N. P. Proukakis, K. Burnett, H. T. C. Stoof, Phys. Rev. A. 57 (1998) 1230.

[15] A. Minguzzi, S. Conti, M. P. Tosi, J. Phys.: Condens. Matter 9 (1997) L33.

[16] S. A. Morgan, M. D. Lee, K. Burnett, Phys. Rev. A. 65 (2002) 022706.

[17] P. Vignolo, A. Minguzzi, M. P. Tosi, Phys. Rev. A. 62 (2000) 023604.

[18] G. Baym, C. J. Pethick, Phys. Rev. Lett. 76 (1996) 6.

[19] See, for instance N. V. Prokof'ev, O. A. Ruebenacker, B. V. Svistunov, Phys. Rev. Lett. 87 (2001) 270402. We stress that the low-momentum cut-off that we have adopted is merely a numerical expedient and does not imply a cut-off of long-wavelength phonons as was assumed by some authors in dealing with a $2 \mathrm{D}$ condensate. For an up-to-date discussion of the latter topic, see the HartreeFock-Bogoliubov treatment of a trapped 2D Bose gas by C. Gies, B. P. van Zyl, S. A. Morgan and D. A. W. Hutchinson, cond-mat/0308177.

[20] F. Dalfovo, S. Giorgini, S. Stringari, Rev. Mod. Phys. 71 (1999) 463.

[21] V. Bagnato, D. Kleppner, Phys. Rev. A. 44 (1991) 7439. 


\section{Figure captions}

Figure 1. The correction term $A(\beta \mu)=\sum_{n=1}^{\infty} \operatorname{Ei}(-n \beta \mu)$ from excited-state occupancy (dashed line) and its approximate form from Eq. (8) (solid line) as functions of $\beta \mu$. In the inset a zoom of the region $0.2<\beta \mu<1$ is shown.

Figure 2. Interaction strengths (in units of $\hbar^{2} / m$ ) as functions of temperature $T$ (in units of ideal-gas critical temperature $T_{0}$ ).

Figure 3. Condensate fraction $N_{0} / N$ as a function of temperature $T$ (in units of $T_{0}$ ) from the $g_{n}^{M B}$ model and (dashed line) compared with the noninteracting gas (solid line).

Figure 4. Density profile of the condensate (solid line) and the thermal cloud (dashed line) in the $g_{n}^{M B}$ model (in units of $N / a_{h o}^{2}$ with $a_{h o}=(\hbar / m \omega)^{1 / 2}$ ) as functions of radial distance $r$ (in units of $a_{h o}$ ) at various values of the temperature $\left(T / T_{0}=0,0.3,0.5,0.8\right.$ and 0.95 from (a) to (e)). 


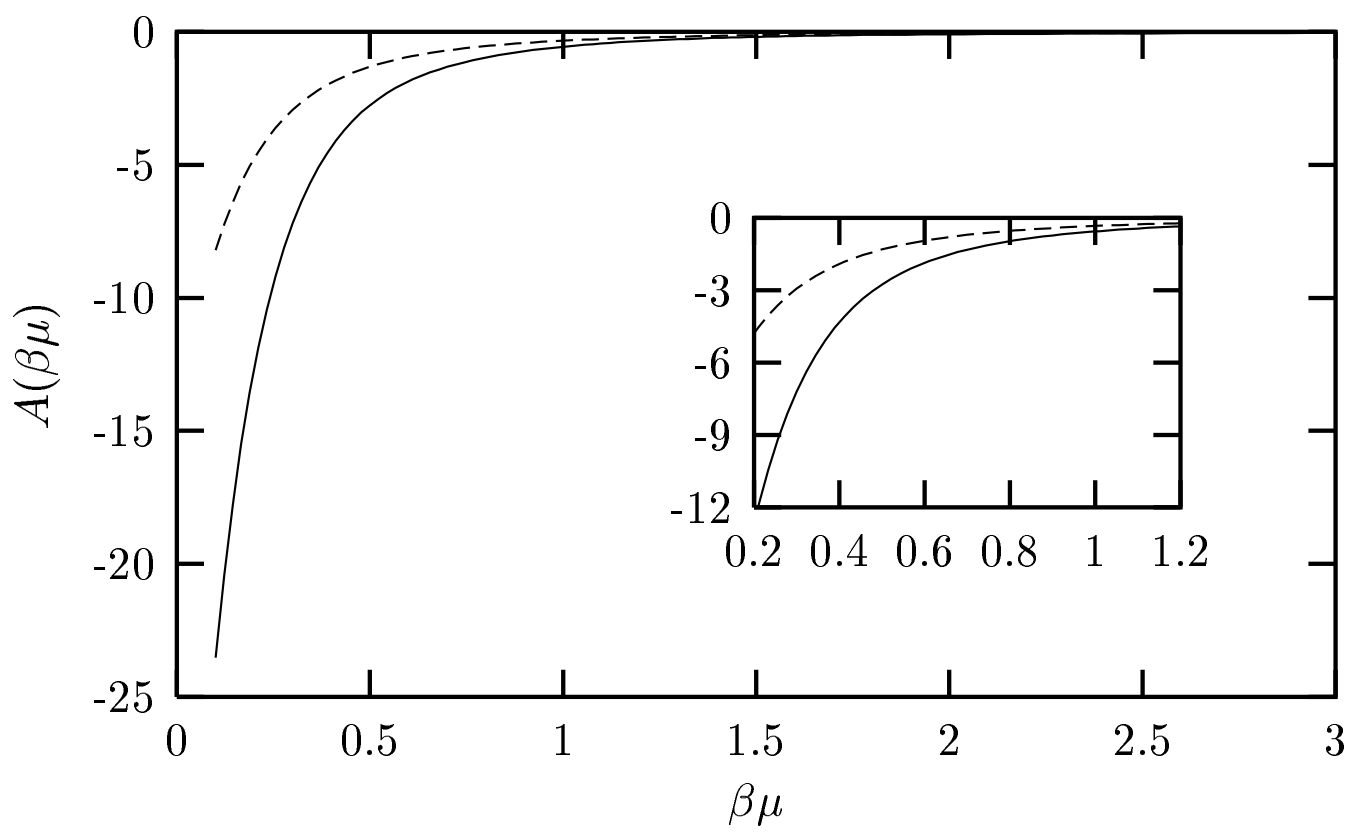

Fig. 1. The correction term $A(\beta \mu)=\sum_{s=1}^{\infty} E i(-s \beta \mu)$ from excited-state occupancy (dashed line) and its approximate form from Eq. (8) (solid line) as functions of $\beta \mu$. In the inset a zoom of the region $0.2<\beta \mu<1.2$ is shown.

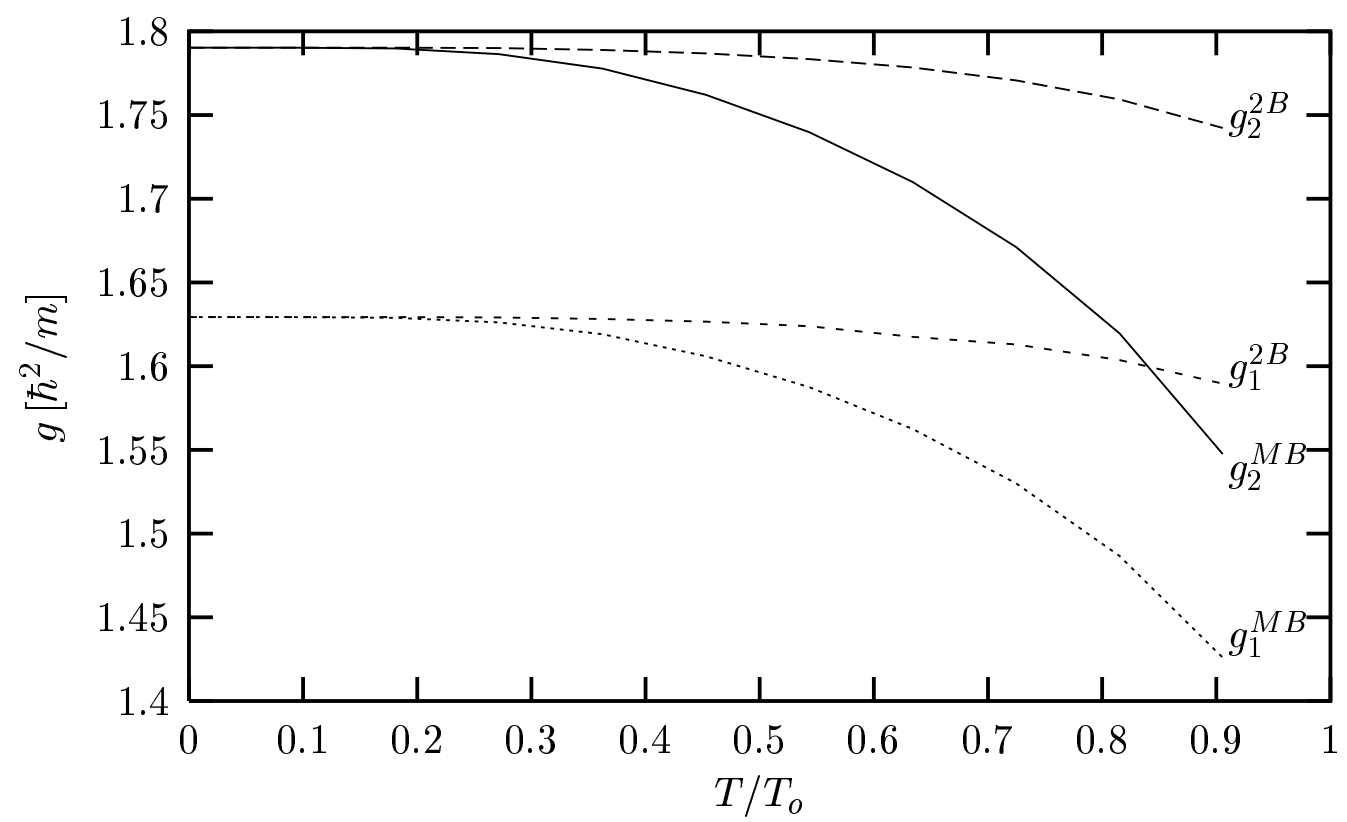

Fig. 2. Interaction strengths (in units of $\hbar^{2} / m$ ) as functions of temperature $T$ (in units of ideal-gas critical temperature $T_{0}$ ). 


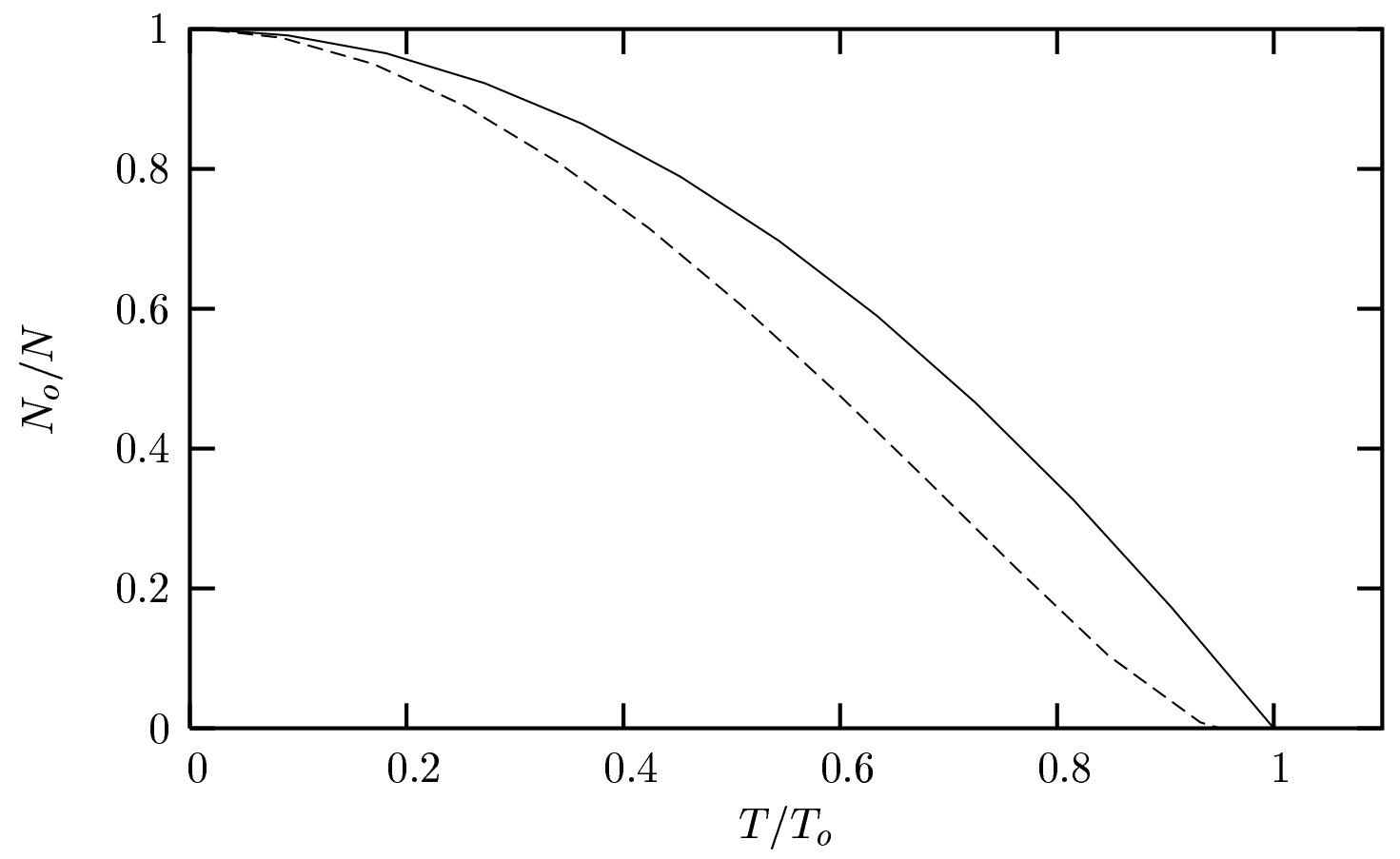

Fig. 3. Condensate fraction $N_{0} / N$ as a function of temperature $T$ (in units of $T_{0}$ ) from the $g_{n}^{M B}$ model and (dashed line) compared with the non-interacting gas (solid line). 


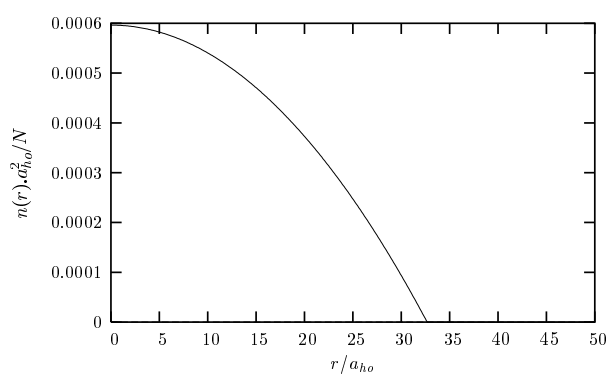

(a)

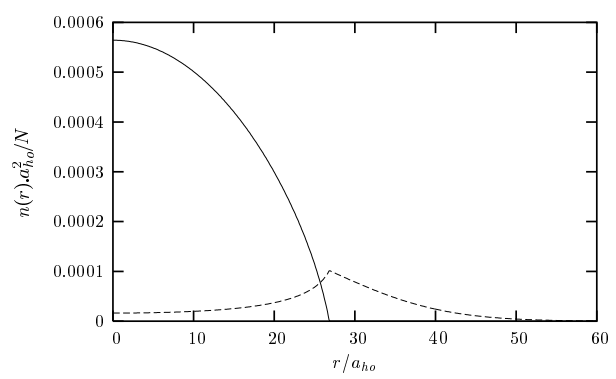

(c)

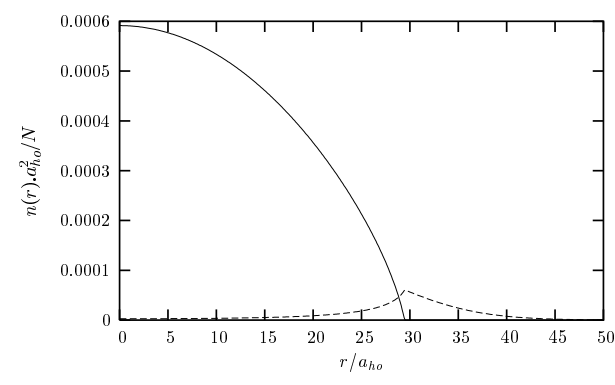

(b)

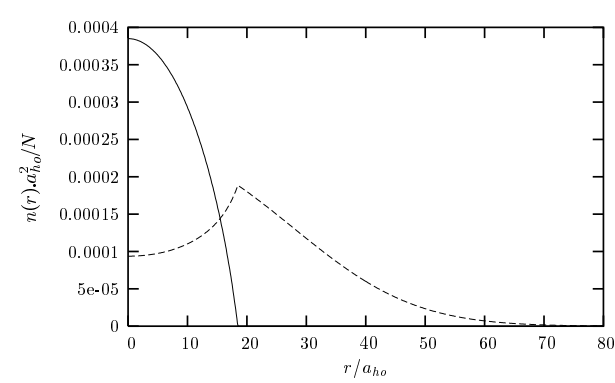

(d)

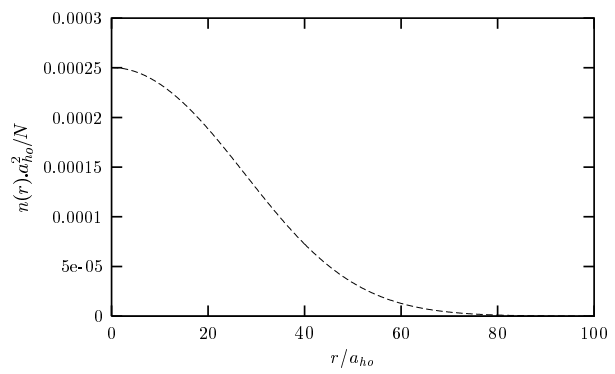

(e)

Fig. 4. Density profile of the condensate (solid line) and the thermal cloud (dashed line) in the $g_{n}^{M B}$ model (in units of $N / a_{h o}^{2}$ with $a_{h o}=(\hbar / m \omega)^{1 / 2}$ ) as functions of radial distance $r$ (in units of $\left.a_{h o}\right)$ at various values of the temperature $\left(T / T_{0}=0\right.$, $0.3,0.5,0.8$ and 0.95 from (a) to (e)). 